Mathematical Research Letters 10, 603-619 (2003)

\title{
MONOTONE QUOTIENTS OF SURFACE DIFFEOMORPHISMS
}

\author{
André de Carvalho and Miguel Paternain
}

\begin{abstract}
A homeomorphism of a compact metric space is tight provided every non-degenerate compact connected (not necessarily invariant) subset carries positive entropy. It is shown that every $C^{1+\alpha}$ diffeomorphism of a closed surface factors to a tight homeomorphism of a generalized cactoid (roughly, a surface with nodes) by a semi-conjugacy whose fibers carry zero entropy.
\end{abstract}

\section{Introduction}

In this article, techniques from point set topology developed in the '20's and '30's and more recent dynamical systems techniques are brought together to construct tight models for surface diffeomorphisms. Roughly speaking, a self-map of a metric space is tight if any non-degenerate continuum behaves chaotically under iteration of the map (i.e. carries positive topological entropy). The tight models of surface diffeomorphisms constructed here are homeomorphisms of singular surfaces (with possibly infinitely many nodes). From the point of view of topological entropy, they retain the dynamically meaningful part of the original maps, and are 'minimal' with respect to this property.

Singular surfaces (a.k.a. surfaces with nodes, generalized cactoids, etc.) and maps between them have been present in mathematics for a long time. They occur naturally in Thurston's theory of surface homeomorphisms. Pseudo-Anosov maps and the generalized pseudo-Anosov maps introduced in $[\mathrm{dC}]$ are examples of tight maps. Thurston's classification theorem for surface homeomorphisms constructs minimal complexity models in each isotopy class of homeomorphisms of the surface. These can be viewed as almost affine maps (i.e. affine maps except for point singularities) on noded Riemann surfaces: by collapsing to points the reducing curves and the finite order components, what remains is a noded Riemann surface and the return map to each piece is a pseudo-Anosov map. These models usually have strictly less dynamics than other maps in the same isotopy class. The tight models constructed here, on the other hand, preserve the dynamics of the original map and collapse to points maximal connected sets which are dynamically irrelevant in the sense of topological entropy. Under appropriate hypotheses, the Thurston and tight models coincide: this is the case, for example, for Axiom A surface diffeomorphisms satisfying some additional hypotheses.

\footnotetext{
Recieved July 19, 2002.
} 
There is some evidence that the class of tight maps on generalized cactoids will provide the framework for the completion of the set of pseudo-Anosov maps and for the construction of almost affine models for surface diffeomorphisms in a quite general context, including Thurston's models as a countable sub-family.

The proofs of the main theorems of the present paper use some technical results about the dynamics on the space of continua. This space may be thought of as a weak version of the tangent bundle and the topological entropy of a continuum as a weak version of the Lyapunov exponent. Although the definition of entropy of non-invariant sets was introduced by Bowen together with his definition of topological entropy, it does not seem to have been used systematically. The results presented in Section 5 use this definition in the context of the space of continua and hold in the general setting of homeomorphisms of compact metric spaces. They are similar in flavor to some results from smooth ergodic theory and may have some independent interest.

In Section 2 the preliminary definitions and the statements of the main theorems (Theorems 1 and 2) are given. Section 3 presents an example: the quotient of Smale's horseshoe under the zero-entropy equivalence. It is is useful to keep it in mind while reading the proofs of the main theorems, which are given in Section 4. The proof of a technical result needed in Section 4 is postponed to Section 5. In Section 6, Theorem 3, giving sufficient conditions for maps to be tight, is proved and Section 7 contains some problems for further research.

\section{Background and statement of results}

Begin by recalling Bowen's definition of topological entropy (see [Bow71, Gro87, KH95]). Let $(X, d)$ be a metric space and $f: X \varsigma$ be a uniformly continuous homeomorphism. Define

$$
d_{n}(x, y)=\max \left\{d\left(f^{i}(x), f^{i}(y)\right) \mid 0 \leq i<n\right\} .
$$

A set $E$ is $(n, \varepsilon)$-separated if for any two distinct points $x, y \in E, d_{n}(x, y)>\varepsilon$. A set $F(n, \varepsilon)$-spans another set $K$ provided that for every $x \in K$ there exists $y \in F$ such that $d_{n}(x, y) \leq \varepsilon$. Let $K \subset X$ be a compact subset and define the following quantities: $r(n, \varepsilon, K)$ is the minimal cardinality of a set which $(n, \varepsilon)$ spans $K, s(n, \varepsilon, K)$ is the maximal cardinality of an $(n, \varepsilon)$-separated subset of $K$ and $D(n, \varepsilon, K)$ is the minimum number of sets whose $d_{n}$-diameter is smaller than $\varepsilon$ and whose union covers $K$. With these definitions, the limits below all exist and are equal and $h(f, K)$ is defined to be equal to all of them:

$$
\begin{aligned}
h(f, K) & =\lim _{\varepsilon \rightarrow 0} \limsup _{n \rightarrow \infty} \frac{1}{n} \ln r(n, \varepsilon, K) \\
& =\lim _{\varepsilon \rightarrow 0} \limsup _{n \rightarrow \infty} \frac{1}{n} \ln s(n, \varepsilon, K) \\
& =\lim _{\varepsilon \rightarrow 0} \lim _{n \rightarrow \infty} \frac{1}{n} \ln D(n, \varepsilon, K)
\end{aligned}
$$


It is not hard to show that $h(f, f(K))=h(f, K)$ and, provided $K$ is $f$-invariant, that $h(f, K)=h\left(f^{-1}, K\right)$. Define the (topological) entropy of $f$ in $K$ or the entropy carried by $K$ under $f$ to be $h_{ \pm}(K)=\max \left\{h(f, K), h\left(f^{-1}, K\right)\right\}$. The topological entropy of $f$ is defined as $h(f)=\sup h(f, K)$, where the supremum is taken over all compact subsets $K \subset X$.

A set is non-degenerate if it contains more than one point. A continuum is a compact connected (subset of a) metric space.

Definition 1. A homeomorphism $f: X \Im$ is tight if $h_{ \pm}(C)>0$ for every nondegenerate continuum $C \subset X$.

Now cactoids and generalized cactoids are defined. This requires several more simple definitions which are given in the list below. Let $X$ be a connected topological space:

a) A point $p \in X$ for which $X \backslash\{p\}$ is not connected, is a cut point of $X$.

b) An endpoint of $X$ is a point which has arbitrarily small neighborhoods whose boundary is a single point.

c) A cut point $q$ separates two points $p, p^{\prime}$ if it is possible to write $X \backslash\{q\}=A \cup B$ where $p \in A, p^{\prime} \in B$ and $\bar{A} \cap B=\emptyset=A \cap \bar{B}$.

d) If $p \in X$ is neither a cut point nor an endpoint of $X$, the set of all points which cannot be separated from $p$ by any other point in $X$ is called a (simple) link of $X$.

Definition 2. Let $X$ be a locally connected continuum. If each simple link of $X$ is homeomorphic to the 2 -sphere $\mathbb{S}^{2}$ then $X$ is called a cactoid. Let $Y$ be a space each link of which is homeomorphic to a surface and all but finitely many links are homeomorphic to $\mathbb{S}^{2}$, and $X$ be obtained by identifying finitely many pairs of points of $Y$. Such an $X$ is called a generalized cactoid (Figure 1).

A generalized cactoid thus may have parts which are graphs or dendrites, but all its 'fat' parts have to be surfaces and, in fact, all but finitely many have to be 2-spheres.

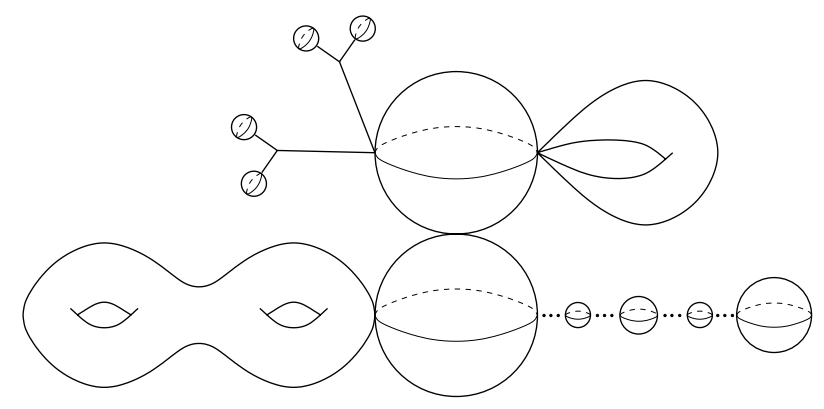

Figure 1. A generalized cactoid. 
A map $f: X \unlhd$ is semi-conjugate to $g$ : $Y \unlhd$ if there is a continuous surjective map $\pi: X \rightarrow Y$ such that $g \circ \pi=\pi \circ f$. The sets of the form $\pi^{-1}(x)$ are the fibers of the semi-conjugacy. If $\pi$ is a homeomorphism, then $f, g$ are conjugate.

The main result of this paper is

Theorem 1. Every $C^{1+\alpha}$ diffeomorphism of a closed surface is semi-conjugate to a tight homeomorphism of a generalized cactoid by a semi-conjugacy whose fibers carry zero entropy.

This theorem is, in fact, a corollary of Theorem 2 below. In order to state it, it is necessary to introduce the following definition, which is central to the paper:

Definition 3. Let $f: X \circlearrowleft$ be a homeomorphism. Define two points $x$ and $y$ to be zero-entropy equivalent if there is a continuum $C$ containing both points with $h_{ \pm}(C)=0$.

That this indeed defines an equivalence relation follows from the facts that $h\left(f, K \cup K^{\prime}\right)=\max \left\{h(f, K), h\left(f, K^{\prime}\right)\right\}$ and that the union of two connected sets with one point in common is also connected.

It is in dimensions 1 and 2 that the zero-entropy equivalence seems to be most interesting, usually inducing a non-trivial partition of the space.

Definition 4. Let $X$ be a metric space and $\mathcal{G}$ a partition (or decomposition) of $X . \mathcal{G}$ is a monotone decomposition if it is a partition into connected sets. It is upper semi-continuous if the sets in $\mathcal{G}$ are compact and for each set $\gamma \in \mathcal{G}$ and each open set $U \supset \gamma$, there exists another open set $V \supset \gamma$ such that every $\gamma^{\prime} \in \mathcal{G}$ intersecting $V$ is contained in $U$.

The collection $\mathcal{G}$ should also be thought of as the collection of equivalence classes of the equivalence relation defined by $x \sim y$ if and only if $x, y \in \gamma$ for some $\gamma \in \mathcal{G}$. If $X$ is a compact metric space, which is the case considered in this paper, then $\mathcal{G}$ is upper semi-continuous if and only if $x_{n} \sim y_{n}, x_{n} \rightarrow x$ and $y_{n} \rightarrow y$ imply $x \sim y$. In what follows, we will refer interchangeably to the equivalence relation and the corresponding decomposition.

Theorem 1 is a consequence of

Theorem 2. Let $f: M \varsigma$ be a $C^{1+\alpha}$ diffeomorphism of a closed surface $M$. Then the zero-entropy equivalence relation induces a monotone upper semi-continuous decomposition of $M$. Moreover, the elements of this decomposition carry zero entropy.

The topological tool used to derive Theorem 1 from Theorem 2 is a theorem by Roberts and Steenrod [RS38], which generalizes for surfaces a result Moore [Moo62] proved for the sphere. Moore's theorem states that, if $\sim$ is a monotone upper semi-continuous equivalence relation on the sphere $\mathbb{S}^{2}$, then the quotient space $\mathbb{S}^{2} / \sim$ is a cactoid; moreover, if no equivalence class of $\sim$ separates $\mathbb{S}^{2}$, then $\mathbb{S}^{2} / \sim$ is homeomorphic to $\mathbb{S}^{2}$. Roberts and Steenrod's theorem 
states that if $M$ is a closed surface and $\sim$ is a monotone upper semi-continuous equivalence relation then the quotient space is a generalized cactoid. (The definition of generalized cactoid given here is slightly more general than the one in [RS38], making their result simpler to state. In their paper, the definition does not include the possibility of identifying finitely many pairs of points as is done in Definition 4.)

Now a theorem giving a sufficient condition for a map to be tight is stated.

Definition 5. A homeomorphism $f: X \unlhd$ of a metric space $X$ is continuumexpansive if there is $\varepsilon>0$ such that, if a continuum $C$ satisfies $\operatorname{diam} f^{n}(C) \leq \varepsilon$ for every $n \in \mathbb{Z}$, then $C$ is a point.

Expansive homeomorphisms (that is, homeomorphisms for which there exists a constant $c>0$ such that iteration under $f$ or $f^{-1}$ brings any two distinct points at least $c$ apart) are clearly continuum-expansive but the converse is not true: pseudo-Anosov homeomorphisms with 1-pronged singularities are continuumexpansive but are not expansive (for example, if $A: \mathbb{T}^{2} \checkmark$ a linear torus Anosov, the quotient space $\mathbb{T}^{2} / \sim$ under the identification $x \sim-x$ is homeomorphic to the 2 -sphere $\mathbb{S}^{2}$ and the projection map $\pi: \mathbb{T}^{2} \rightarrow \mathbb{S}^{2}$ is a branched covering with 4 branch points; the map $A$ induces a map $f_{A}: \mathbb{S}^{2} \Im$ which is a pseudo-Anosov map with 1-pronged singularities at the branch points.)

A partial characterization of tight maps is given by

Theorem 3. Continuum-expansive homeomorphisms are tight.

\section{An example}

Now a brief description of the zero-entropy equivalence relation for Smale's horseshoe map ([Sma67]) is given. For a more detailed discussion of this and other related examples, see $[\mathrm{dC}]$.

The horseshoe is a homeomorphism $f: \mathbb{S}^{2} \unlhd$ which stretches horizontally and squeezes vertically a stadium shaped region $R$, placing it inside itself as shown in Figure 2. There is a repeller at infinity whose basin contains all points outside $R$. The horseshoe has two saddle fixed points which are labeled $x_{0}$ and $x_{1}$ (shown as $\bullet$ and $\circ$, respectively, in Figure 2) and an attracting fixed point in the shaded semi-circular region on the left denoted by $x$ (shown as $\mathbf{\square}$ ).

Denote by $\mathcal{H}^{u}$ and $\mathcal{H}^{s}$ the closures of the unstable and stable manifolds of the fixed point $x_{0}$ (or indeed of any other periodic point, since their closures coincide) and let $\mathcal{H}=\mathcal{H}^{s} \cup \mathcal{H}^{u}$. Equivalence classes of the zero-entropy equivalence for the horseshoe are of four kinds:

a) Closures of connected components of $\mathbb{S}^{2} \backslash \mathcal{H}$.

b) Closures of connected components of $\mathcal{H}^{u} \backslash \mathcal{H}^{s}$ (not already contained in sets in a)).

c) Closures of connected components of $\mathcal{H}^{s} \backslash \mathcal{H}^{u}$ (not already contained in sets in a)).

d) Single points which are in none of the sets in a), b) or c). 


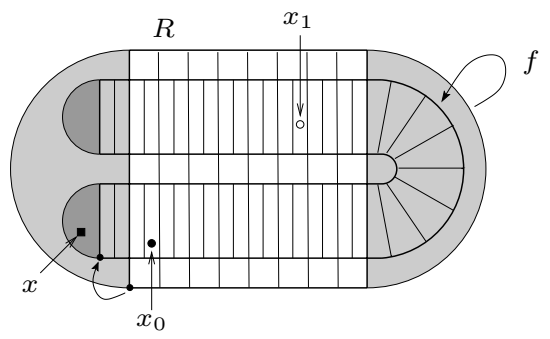

Figure 2. The horseshoe map.

To see that these sets do not carry entropy, notice that all points in any connected component of $\mathbb{S}^{2} \backslash \mathcal{H}$ (before taking the closure) converge to the attracting fixed point $x$. It is not hard to see that, after taking the closure nothing more significant happens and this shows the sets in a) indeed carry no entropy. The same holds for sets of types b) and c). To see that any larger continuum must contain entropy, notice that if $C$ is a connected set that contains two distinct sets among the ones described above, then it must intersect a Cantor set's worth of invariant manifolds, either stable or unstable (or both). It follows that at least one of its $\omega$ - or $\alpha$-limit sets contains all the non-wandering set of the horseshoe and therefore one of $h(f, C)$ or $h\left(f^{-1}, C\right)$ equals $\ln 2$.

The quotient space is represented in Figure 3. It is again a sphere, obtained by identifying the solid boundary in the figure along the dotted arcs from the mid-point at the top to the corner point on the lower left. The stable and unstable manifolds of the horseshoe project to two transverse foliations with singularities, represented by solid and dashed lines, respectively. In fact, these foliations carry transverse invariant measures whose product gives a Euclidean structure on the sphere. The quotient map preserves both foliations, dividing one of the transverse measures by 2 and multiplying the other also by 2 , so that the product measure is invariant. This map is a generalized pseudo-Anosov map (see $[\mathrm{dC}])$.

\section{Proofs of Theorems 1 and 2}

The idea of the proof is roughly as follows. It is necessary to show that carrying zero entropy is a closed condition or, alternatively, that carrying positive entropy is an open condition: if a continuum $C$ carries (positive) entropy, then all nearby continua must also carry entropy. Suppose then that $h_{ \pm}(C)>0$. The first step is to obtain, using the dynamics on the space of continua, an ergodic invariant measure with positive entropy such that arbitrarily near almost every point there is an iterate of $C$ with definite diameter. In the context of $C^{1+\alpha}$ surface diffeomorphisms, results due to Katok [Kat80, KH95] show that there must be a horseshoe near the support of any ergodic measure with positive entropy. This implies that iterates of $C$ must eventually intersect the (un)stable set of this horseshoe. The 2-dimensionality of the ambient space forces such 


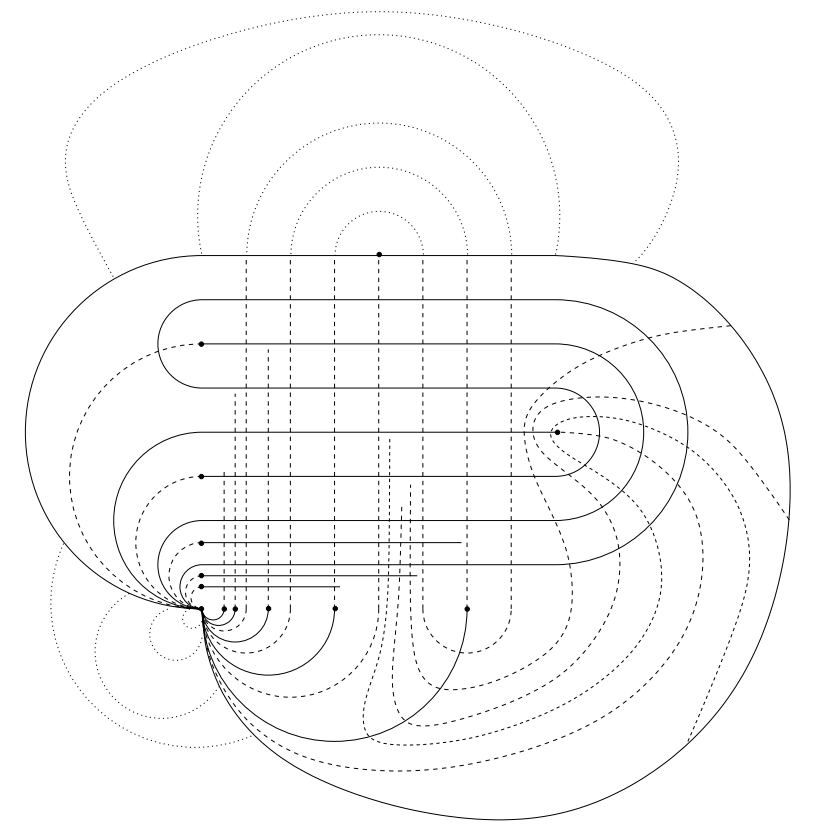

FiguRE 3. The quotient of the sphere under the zero-entropy equivalence relation for the horseshoe.

intersections to be 'transversal,' so that (appropriate iterates of) continua near $C$ must also intersect the (un)stable set of the horseshoe and thus carry entropy.

Begin by setting some definitions and notations. Let $(X, d)$ be a metric space and $\mu$ a Borel probability measure $(B p m)$ on $X$. The support of $\mu, \operatorname{supp} \mu$, is the set of points $x \in X$ such that every neighborhood $V \ni x$ satisfies $\mu(V)>0$. A point $x \in X$ is an atom ${ }^{1}$ of $\mu$ if $\mu(\{x\}) \neq 0$.

The Hausdorff distance between two compact subsets $A, B \subset X$ is

$$
d_{H}(A, B)=\max \left\{\max _{a \in A} d(a, B), \max _{b \in B} d(b, A)\right\}
$$

where, if $x \in X, C \subset X, d(x, C)=\inf _{c \in C} d(x, c)$. If $X$ is compact, the Hausdorff metric makes the set $\mathcal{K}=\mathcal{K}(X)$ of all compact subsets of $X$ into a compact metric space.

The following lemma is one of the most important technical ingredients in the proof of the main theorems. Its proof is given in the next section. Let $f: X \varsigma$ be a homeomorphism and denote by $\omega(C) \subset \mathcal{K}$ the $\omega$-limit set of $C \in \mathcal{K}$ with respect to the map $K \mapsto f(K)$. If $\mu$ is an $f$-invariant Bpm, $h_{\mu}(f)$ denotes the measure theoretic entropy of $f$ with respect to $\mu$.

\footnotetext{
${ }^{1}$ This definition applies to Borel measures in separable metric spaces.
} 
Lemma 4. Let $(X, d)$ be a compact metric space, $f: X \unlhd$ be a homeomorphism and $C \subset X$ be a compact set satisfying $h(f, C)>0$. Then there exists an $f$ invariant ergodic Bpm $\mu$ on $X$, such that $h_{\mu}(f)>0$ and such that $\mu$-almost every point belongs to a non-degenerate set in $\omega(C)$.

Now the assumption of differentiability is added to the discussion and attention is restricted to surfaces. Although the assumption that $f$ be $C^{1}$ is all that is needed for some of the definitions and results listed below, in order to use Pesin Theory, which is essential to our results, it is necessary to assume some extra regularity: from now on, $M$ will denote a compact smooth Riemannian surface without boundary and $f$ : $M \lessgtr a C^{1+\alpha}$ diffeomorphism, with $0<\alpha \leq 1$.

The results now described follow from Pesin Theory [Pes76, PS89, Kat80, KH95]. The concepts needed are quite technical and the definitions rather involved. Instead of presenting them in detail, which would duplicate what is contained in the papers cited, only a description of the concepts and results that will be used is given.

An $f$-invariant probability measure $\mu$ is hyperbolic if all the Lyapunov exponents of $f$ are non-zero at $\mu$-almost every point. Notice that if $\mu$ is an ergodic $f$-invariant Bpm on (the surface) $M$ with $h_{\mu}(f)>0$ then ergodicity implies that $\mu$ has no atoms and Ruelle's inequality implies that it is hyperbolic. This observation will be used below.

Assume that $\mu$ is a non-atomic hyperbolic ergodic $f$-invariant Bpm. Given $0<\delta<1$ there exists a compact Pesin set $\Lambda_{\delta}$ with $\mu\left(\Lambda_{\delta}\right)>1-\delta$ and with the properties that are now described. To avoid cluttering the notation, the dependence on $\delta$ and the point $p$ is not incorporated into it. For every $p \in \Lambda_{\delta}$ there exist an open neighborhood $N \ni p$, a compact sub-neighborhood $V \ni p$, and a diffeomorphism $\Psi:(-1,1)^{2} \rightarrow N$, with $\Psi(0,0)=p$ and $\Psi\left([-1 / 10,1 / 10]^{2}\right)=V$, such that the $N$-local unstable manifolds $W_{N}^{u}(y)$ of all points $y$ in $\Lambda_{\delta} \cap V$ are the images under $\Psi$ of graphs of the form $\{(v, \varphi(v)) \mid v \in(-1,1)\}$ with small Lipschitz constant. Any two such local unstable manifolds are either disjoint or equal and they depend continuously on the point $y \in \Lambda_{\delta} \cap V$. Similarly, the $N$-local stable manifolds $W_{N}^{s}(y)$ of points $y$ in $\Lambda_{\delta} \cap V$ are the images under $\Psi$ of graphs of the form $\{(\varphi(v), v) \mid v \in(-1,1)\}$ with small Lipschitz constant. Any two such local unstable manifolds are either disjoint or equal and they depend continuously on the point $y \in \Lambda_{\delta} \cap V$. Stable and unstable manifolds of points in $V$ having the properties just described are called $N$-admissible.

It follows that there is a continuous product structure: given any $x, y \in \Lambda_{\delta} \cap V$, the intersection $W_{N}^{u}(x) \cap W_{N}^{s}(y)$ is transversal and consists of exactly one point, which is denoted by $[x, y]$. Define maps $\pi_{x}^{s}: \Lambda_{\delta} \cap V \rightarrow W_{N}^{s}(x)$ and $\pi_{x}^{u}: \Lambda_{\delta} \cap V \rightarrow$ $W_{N}^{u}(x)$ by $\pi_{x}^{s}(y)=[y, x]$ and $\pi_{x}^{u}(y)=[x, y]$. Observe, however, that $[x, y]$ need not be in $\Lambda_{\delta}$.

Katok's Closing Lemma (the Main Lemma in [Kat80]) will also be needed: it states that arbitrarily near any recurrent point in $\Lambda_{\delta} \cap V$ there exist hyperbolic periodic saddles whose invariant manifolds are $N$-admissible. 
Let $A$ denote the subset of $\Lambda_{\delta} \cap V$ consisting of all points which are both forward and backward recurrent. By the Poincaré Recurrence Theorem, $\mu\left(\left(\Lambda_{\delta} \cap\right.\right.$ $V) \backslash A)=0$.

Definition 6. A point $x \in A$ is $\delta$-inaccessible or simply inaccessible if it is accumulated on both sides of $W_{N}^{s}(x)$ by points in $\pi_{x}^{s}(A)$ and is accumulated on both sides of $W_{N}^{u}(x)$ by points in $\pi_{x}^{u}(A)$. Otherwise, $x$ is accessible.

Notice that this definition does depend on the choices made.

By a rectangle it is meant a Jordan curve made up of alternating segments of stable and unstable manifolds, two of each. The segments forming the boundary are its sides and the intersection points of the sides are the corners. A rectangle is said to enclose $p$ if it is the boundary of an open topological disk containing $p$.

Lemma 5. Let $x \in A$ be an inaccessible point. Then there exist rectangles enclosing $x$, having sides along the invariant manifolds of hyperbolic periodic saddles in $V$ and having arbitrarily small diameter.

Proof. By assumption, there are points in $A$ whose stable manifolds are arbitrarily near that of $x$ and to the right of it (think of stable manifolds as roughly vertical and unstable manifolds as roughly horizontal). Since points in $A$ are recurrent, by Katok's Closing Lemma, it is possible to find a periodic point, also to the right of the stable manifold of $x$, whose stable manifold is $N$-admissible. Proceeding like this, it is possible to find periodic points, whose invariant manifolds are $N$-admissible, on all four 'quadrants' determined by the stable and unstable manifolds of $x$. Clearly, segments of these manifolds form a rectangle enclosing $x$ and the choices can be made so that the rectangle has arbitrarily small diameter.

Notice it now follows from standard arguments that these periodic saddles have transverse homoclinic intersections. In particular, the closures of their invariant manifolds carry positive entropy. This observation will be used below.

Lemma 6. The set of accessible points in $A$ has measure 0.

Proof. Pick $x \in A$ and define $L^{s} \subset W_{N}^{s}(x)$ to be the set of endpoints of the non-degenerate components of $W_{N}^{s}(x) \backslash \pi_{x}^{s}(A)$ and $L^{u} \subset W_{N}^{u}(x)$ to be the set of endpoints of the non-degenerate components of $W_{N}^{u}(x) \backslash \pi_{x}^{u}(A)$. Then both $L^{s}, L^{u}$ are countable and it follows from the definitions that the set of accessible points in $A$ lie on $\left(\pi_{x}^{s}\right)^{-1}\left(L^{s}\right) \cup\left(\pi_{x}^{u}\right)^{-1}\left(L^{u}\right)$. Since each fiber of $\pi_{x}^{s}, \pi_{x}^{u}$ contains at most one point which is both forward and backward recurrent and since $\mu$ is non-atomic, it follows that the sets $\left(\pi_{x}^{s}\right)^{-1}\left(L^{s}\right),\left(\pi_{x}^{u}\right)^{-1}\left(L^{u}\right)$, and thus the set of accessible points, have measure 0 .

In the proof below the following concept will be used. Let $\gamma$ be an open arc. A connected set $C$ is said to cross $\gamma$ if there exist two disjoint connected subsets $C_{1}, C_{2} \subset C \backslash \gamma$, on different sides $^{2}$ of $\gamma$, and a (possibly degenerate) sub-arc

\footnotetext{
${ }^{2}$ In order to make precise sense of the 'different sides of $\gamma$ ' one has to invoke the Jordan Curve Theorem.
} 


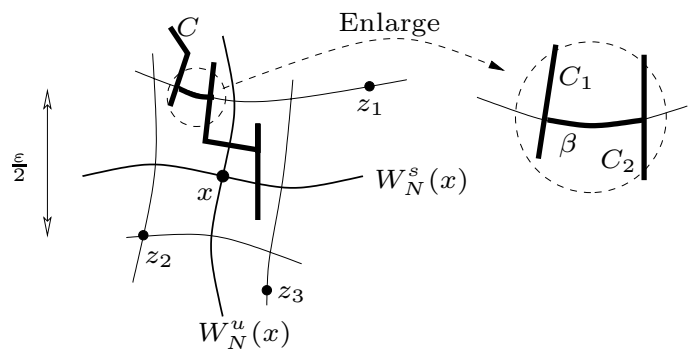

FiguRE 4. An inaccessible point and a nearby continuum.

$\beta \subset \gamma \cap C$, such that $C_{1} \cup \beta \cup C_{2}$ is connected (see the diagram on the right in Figure 4).

Lemma 7. Suppose $x \in A$ is an inaccessible point. Given $\varepsilon>0$, there exists $0<r<\varepsilon$ such that, if $z, w$ are any two points satisfying $d(x, z)<r$ and $d(x, w)>\varepsilon$, then $z, w$ are not zero-entropy equivalent.

Proof. By Lemma 5, there exists a rectangle $R$ enclosing $x$ with $\operatorname{diam}(R)<$ $\varepsilon$ and with sides along the invariant manifolds of periodic points. Moreover, by the observation following the proof of that lemma, these periodic points have transverse homoclinic intersections and, therefore, their invariant manifolds carry entropy. Pick $0<r<d(x, \partial R)$ (so that $r<\varepsilon$ since $R$ is a Jordan curve enclosing $x$ ). If $z, w$ are points satisfying the hypotheses of the lemma and $C$ is a continuum containing $z, w$ then $C$ must cross the boundary of $R$ (see Figure 4 where $z_{i}, i=1,2,3$ are periodic points). The proof of the lemma now follows from the simple observations below:

a) If a continuum $C$ crosses the stable manifold of a periodic saddle $y$ of period $k$, then there is a compact subset $K \subset C$ such that $d_{H}\left(f^{n k}(K), \overline{W^{u}(y)}\right) \rightarrow 0$ as $n \rightarrow \infty$ (and an analogous statement holds exchanging the roles of stable and unstable manifolds).

b) If $K, K^{\prime}$ are compact sets and $d_{H}\left(f^{n}(K), f^{n}\left(K^{\prime}\right)\right) \rightarrow 0$ as $n \rightarrow \infty$, then $h(f, K)=h\left(f, K^{\prime}\right)$.

Lemma 8. If $C$ is a continuum with $h_{ \pm}(C)>0$ then every continuum that is close enough to $C$ in the Hausdorff metric contains inequivalent points. In particular, carrying positive entropy is an open condition in the space of continua.

Proof. Assume that $h(f, C)>0$ : an analogous argument can applied to $f^{-1}$ in case $h\left(f^{-1}, C\right)>0$. Let $\mu$ be the measure given by Lemma 4 . Just as before, it follows that $\mu$ is non-atomic and hyperbolic. Choose $0<\delta<1, \Lambda_{\delta}, p$ and $V$ as in the discussion of Pesin Theory above so that $\mu(A)>0$, where $A$ is the set of recurrent points in $\Lambda_{\delta} \cap V$. By Lemmas 4 and 6 , there exists an inaccessible point $x \in A$ lying on a non-degenerate continuum $E \in \omega(C)$. Let $\varepsilon=\operatorname{diam}(E) / 10>$ 0 and let $0<r<\varepsilon$ be given by Lemma 7. Since $E \in \omega(C)$, there exist 
$\rho>0$ and $k \in \mathbb{N}$ such that, if $D$ is any continuum with $d_{H}(D, C)<\rho$, then $d_{H}\left(f^{k}(D), E\right)<r$. Since $x \in E$ this implies that there exist points $z, w \in f^{k}(D)$ such that $d(z, x)<r$ and $d(w, x)>\varepsilon$. For the latter inequality, notice that there is $y \in E$ such that $d(x, y) \geq \operatorname{diam}(E) / 2=5 \varepsilon$ and that there is $w \in f^{k}(D)$ such that $d(w, y)<r<\varepsilon$. Thus, $d(w, x) \geq d(x, y)-d(w, y)>5 \varepsilon-\varepsilon=4 \varepsilon$. It now follows from Lemma 7 that $0<h_{ \pm}\left(f^{k}(D)\right)=h_{ \pm}(D)$.

Proof of Theorem 2. Monotonicity is obvious from the definition of zero-entropy equivalence. Upper semi-continuity now follows easily from Lemma 8: if $x_{n} \rightarrow x$, $y_{n} \rightarrow y$ and $C_{n}$ are continua containing both $x_{n}, y_{n}$ with $h_{ \pm}\left(C_{n}\right)=0$, by compactness there is a convergent subsequence $C_{n_{k}} \rightarrow C$, where $C$ is a continuum, $C \ni x, y$ and, by Lemma $8, h_{ \pm}(C)=0$. That the equivalence classes carry zero entropy also follows immediately from Lemma 8.

Proof of Theorem 1. Let $M_{\sim}$ denote the quotient of $M$ under the zero-entropy equivalence. By the Moore-Roberts-Steenrod theorems, $M_{\sim}$ is a generalized cactoid. Let $\pi: M \rightarrow M_{\sim}$ denote the quotient map. Since the zero-entropy equivalence is clearly $f$-invariant, $f$ projects to a homeomorphism $f_{\sim}: M_{\sim} \leftrightarrows$. To finish the proof of the theorem all there is left is to show is that $f_{\sim}$ is tight. The proof of Theorem 17 in [Bow71] shows that for any compact set $C \subset M_{\sim}$

$$
h\left(f, \pi^{-1}(C)\right) \leq h\left(f_{\sim}, C\right)+\sup _{x \in C} h\left(f, \pi^{-1}(x)\right) .
$$

Since the fibers of $\pi$ do not carry entropy, it follows that $h\left(f, \pi^{-1}(C)\right) \leq$ $h\left(f_{\sim}, C\right)$. If $C \subset M_{\sim}$ is a non-degenerate continuum, then $\pi^{-1}(C)$ is also a continuum (since $\pi$ is monotone) and contains inequivalent points (since $C$ is non-degenerate). Therefore $h\left(f, \pi^{-1}(C)\right)>0$, which completes the proof.

\section{Proof of Lemma 4}

The lemmas below, whose proofs can be found in [KH95] (Lemma 4.5.2) and [Mn87] (Lemma 11.8) respectively, will be central to what follows.

Lemma 9. Let $X$ be a compact metric space, $f: X \unlhd$ a homeomorphism, $E_{n}$ $(n, \varepsilon)$-separated sets,

$$
\nu_{n}=\frac{1}{\operatorname{card}\left(E_{n}\right)} \sum_{p \in E_{n}} \delta_{p}
$$

the uniform $\delta$-measures on $E_{n}$ and

$$
\mu_{n}=\frac{1}{n} \sum_{i=0}^{n-1} f_{*}^{i} \nu_{n} .
$$

Then there exists an accumulation point $\mu$ of $\left\{\mu_{n}\right\}_{n \in \mathbb{N}}$ in the weak* topology such that $\mu$ is an $f$-invariant Bpm satisfying

$$
h_{\mu}(f) \geq \limsup _{n \rightarrow \infty} \frac{1}{n} \ln \operatorname{card}\left(E_{n}\right) .
$$


Lemma 10 (Pliss). For every $\lambda \in \mathbb{R}, \eta>0, H>0$ there are $N_{0}=N_{0}(\lambda, \eta, H) \in$ $\mathbb{N}, \delta=\delta(\lambda, \eta, H)>0$ such that if $a_{1}, \ldots, a_{N}$ are real numbers, $N \geq N_{0},\left|a_{n}\right| \leq$ $H$, and

$$
\sum_{i=1}^{N} a_{i} \leq N \lambda
$$

then there are $1 \leq n_{1}<n_{2}<\ldots<n_{\ell} \leq N$ such that

$$
\sum_{i=n_{j}+1}^{n} a_{i} \leq\left(n-n_{j}\right)(\lambda+\eta)
$$

for $j=1, \ldots, l n_{j}<n \leq N$. Moreover $\ell / N \geq \delta$.

Recall that $\mathcal{K}$ denotes the space of compact subsets of the compact metric space $X$ with the Hausdorff metric. A continuous map (resp. homeomorphism) $f: X \Im$ induces a continuous map (resp. homeomorphism) $\hat{f}: \mathcal{K} \circlearrowleft$ in the obvious way: $\hat{f}(K)=f(K)$. (It may seem pedantic to differentiate between $f$ and $\hat{f}$, but it is useful to do so in order to avoid confusion below.) Recall that $D(n, \varepsilon, K)$ denotes the minimum number of sets with $d_{n}$-diameter less than $\varepsilon$ needed to cover $K$. Notice that, as a function of $K$ (with values in $\mathbb{N}$ ), $D(n, \varepsilon, K$ ) is upper semi-continuous. The following useful inequality is an easy consequence of the definition:

$$
D(m+n, \varepsilon, K) \leq D(m, \varepsilon, K) \cdot D\left(n, \varepsilon, f^{m}(K)\right) .
$$

Define three Borel measurable functions $h_{n, \varepsilon}, h_{\varepsilon}, h: \mathcal{K} \rightarrow[0, \infty]$ by

$$
\begin{aligned}
h_{n, \varepsilon}(K) & =\frac{1}{n} \ln D(n, \varepsilon, K) \\
h_{\varepsilon}(K) & =\lim _{n \rightarrow \infty} h_{n, \varepsilon}(K) \\
h(K) & =\lim _{\varepsilon \rightarrow 0} h_{\varepsilon}(K)
\end{aligned}
$$

Notice that $h(K)=h(f, K)$. That the limit as $n \rightarrow \infty$ exists follows from inequality (1) and that the limit as $\varepsilon \rightarrow 0$ exists follows from the monotonicity of $h_{n, \varepsilon}$ as a function of $\varepsilon$. Clearly, for any $K \in \mathcal{K}, h_{n, \varepsilon}(K) \leq h_{n, \varepsilon}(X)$. As has already been observed, $h$ is $\hat{f}$-invariant, that is, for any compact set $K \in \mathcal{K}$, $h(K)=h(\hat{f}(K))$.

Let $C \in \mathcal{K}$ and define $\mathfrak{M}_{C}$ to be the set of $\hat{f}$-invariant Bpm's $\hat{\mu}$ on $\mathcal{K}$ which are weak $*$ limit points of the set of orbital averages of the form

$$
\hat{\mu}_{n}=\frac{1}{n} \sum_{i=0}^{n-1} \delta_{\hat{f}^{i}(C)}
$$

where $\delta_{x}$ denotes the Dirac mass at the point $x$. Observe that measures in $\mathfrak{M}_{C}$ have support contained in $\omega(C)=\omega_{\hat{f}}(C)$, the $\omega$-limit set of $C$ under $\hat{f}$. 
Lemma 11. Let $X$ be a compact metric space and $f: X \unlhd$ be a homeomorphism and assume $C \in \mathcal{K}$ satisfies $h(C)>0$. Then

$$
\int_{\mathcal{K}} h d \hat{\mu}>0
$$

for every $\hat{\mu} \in \mathfrak{M}_{C}$

Proof. Take $0<\eta<h(C) / 2$ and set $\lambda=-h(C)+\eta$. Choose $\varepsilon_{0}>0$ and $N_{1}$ such that

$$
\ln D\left(n, \varepsilon_{0}, C\right) \geq-\lambda n>0
$$

for every $n \geq N_{1}$. Let $\Gamma_{m}$ denote the set of $K \in \mathcal{K}$ such that $h_{m, \varepsilon_{0}}(K) \geq-\lambda-\eta$. Observe that, by upper semi-continuity, the sets $\Gamma_{m}$ are closed, and therefore compact.

To make the notation less cumbersome, set $c_{n}=D\left(n, \varepsilon_{0}, C\right)$. Set also $c_{0}=1$ and

$$
a_{n}=\ln \frac{c_{n-1}}{c_{n}} .
$$

It follows from inequality (1) that there is $H=H\left(\varepsilon_{0}\right)$, independent of $n$, such that $\left|a_{n}\right| \leq H$.

Observe that

$$
\sum_{i=1}^{N} a_{i}=\ln \frac{c_{0}}{c_{N}}=-\ln c_{N} \leq \lambda N
$$

for every $N \geq N_{1}$. Choose $\delta=\delta\left(\lambda, \eta, \varepsilon_{0}\right)>0$ and $N_{0} \geq N_{1}$ according to Lemma 10 for $\lambda, \eta$ and $H$ as above. We are going to show that, for orbital average measures $\hat{\mu}_{N}$ defined by equality $(2), \hat{\mu}_{N}\left(\Gamma_{m}\right) \geq \frac{\delta}{2}$ for all integers $m$ and $N \geq N_{0}$ such that $m / N<\delta / 2$.

Take $1 \leq n_{1}<n_{2}<\cdots<n_{l} \leq N$ according to Lemma 10 such that

$$
\ln \frac{c_{n_{j}}}{c_{n}}=\sum_{i=n_{j}+1}^{n} a_{i} \leq(\lambda+\eta)\left(n-n_{j}\right)
$$

for $j=1, \ldots, \ell, n_{j}<n \leq N$. Inequality (1) states that $c_{n} \leq c_{n_{j}} \cdot D(n-$ $\left.n_{j}, \varepsilon_{0}, f^{n_{j}}(C)\right)$, from which it follows $h_{n, \varepsilon_{0}}\left(\hat{f}^{n_{j}}(C)\right) \geq-\lambda-\eta$, for $0 \leq n \leq N-n_{j}$.

Write $\ell=k_{1}+k_{2}$, where $k_{1}$ is the number of values $n_{j}$ such that $0 \leq n_{j} \leq$ $N-m$. Then,

$$
\hat{\mu}_{N}\left(\Gamma_{m}\right) \geq \frac{k_{1}}{N}
$$

On the other hand, by Lemma 10,

$$
\frac{k_{1}+k_{2}}{N} \geq \delta
$$

and it follows that

$$
\hat{\mu}_{N}\left(\Gamma_{m}\right) \geq \delta-\frac{m}{N} \geq \frac{\delta}{2} .
$$


It now follows from weak $*$ convergence and from the fact that $\Gamma_{m} \subset \mathcal{K}$ is compact that $\hat{\mu}\left(\Gamma_{m}\right) \geq \delta / 2$, so that

$$
\int h_{m, \varepsilon} d \hat{\mu} \geq \int h_{m, \varepsilon_{0}} d \hat{\mu} \geq(-\lambda-\eta) \frac{\delta}{2} .
$$

As was pointed out above, $h_{m, \varepsilon}$ is uniformly bounded by $h_{m, \varepsilon}(X) \rightarrow h_{\varepsilon}(X)<$ $\infty$. Applying the Dominated Convergence Theorem as $m \rightarrow \infty$ and then the Monotone Convergence Theorem as $\varepsilon \rightarrow 0$, it follows that

$$
\int h d \hat{\mu} \geq(-\lambda-\eta) \frac{\delta}{2}>0
$$

as was claimed.

Denote by $\mathcal{F}$ the set of pairs $(p, C)$ such that $C \in \mathcal{K}$ and $p \in C$. Clearly $\mathcal{F}$ is a compact subset of $X \times \mathcal{K}$. A homeomorphism $f: X \Im$ induces a map $\tilde{f}: \mathcal{F} \circlearrowleft$ setting $\tilde{f}(p, C)=(f(p), \hat{f}(C))$. Let $\pi: \mathcal{F} \rightarrow X$ and $\Pi: \mathcal{F} \rightarrow \mathcal{K}$ denote the canonical projections. As usual with projections, the induced push-forward maps $\pi_{*}$ and $\Pi_{*}$ send ergodic measures to ergodic measures.

It is now possible to give a

Proof of Lemma 4. Let $\hat{\lambda}$ be an ergodic Bpm on $\mathcal{K}$ such that $h>0$ almost everywhere with respect to $\hat{\lambda}$ and supp $\hat{\lambda} \subset \omega(C)$. That such a measure exists follows from the previous lemma and the Ergodic Decomposition Theorem (Theorems II.6.1 and II.6.4 in [Mn87]). From these, it also follows that it is possible to choose a generic point $A$ of the measure $\hat{\lambda}$ (that is, a compact $A \in \mathcal{K}$ such that $\hat{\lambda}$ is the weak $*$ limit of $\left.(1 / n) \sum_{i=0}^{n-1} \delta_{\hat{f}^{i}(A)}\right)$, with $h(A)>0$.

Choose $\varepsilon>0$ and maximal $(n, \varepsilon)$-separated sets $E_{n} \subset A$ such that

$$
\limsup _{n \rightarrow \infty} \frac{1}{n} \ln \operatorname{card}\left(E_{n}\right)>0
$$

and consider the measures on $\mathcal{F}$ given by

$$
\tilde{\nu}_{n}=\frac{1}{\operatorname{card}\left(E_{n}\right)} \sum_{x \in E_{n}} \delta_{(x, A)} \quad \text { and } \quad \tilde{\lambda}_{n}=\frac{1}{n} \sum_{i=0}^{n-1} \tilde{f}_{*}^{i} \nu_{n} .
$$

Setting $\lambda_{n}=\pi_{*}\left(\tilde{\lambda}_{n}\right)$, Lemma 9 implies that there exists a weak $*$ convergent subsequence $\lambda_{n_{k}} \rightarrow \lambda$ so that $h_{\lambda}(f)>0$. Now, let $\tilde{\lambda}$ be a weak $*$ limit of $\left\{\tilde{\lambda}_{n_{k}}\right\}$. Notice that $\pi_{*}(\tilde{\lambda})=\lambda$ and $\Pi_{*}(\tilde{\lambda})=\hat{\lambda}$. Let $N=\{x \in X \mid x$ belongs to a non-degenerate set in $\omega(C)\}$. We claim that $\lambda(N)=1$. To see why this is so, let $\hat{N}=\{K \in \mathcal{K} \mid K \in \omega(C)$ is a non-degenerate set $\}$ and $\tilde{N}=\Pi^{-1}(\hat{N})=$ $\{(x, K) \in \mathcal{F} \mid K \in \omega(C)$ is a non-degenerate set $\}$. Notice that $N=\pi(\tilde{N})$. Since $h>0 \hat{\lambda}$-almost everywhere, $\hat{\lambda}(\hat{N})=1$, so that $\tilde{\lambda}(\tilde{N})=\tilde{\lambda}\left(\Pi^{-1}(\hat{N})\right)=\Pi_{*} \tilde{\lambda}(\hat{N})=$ $\hat{\lambda}(\hat{N})=1$. On the other hand, $\lambda(N)=\pi_{*} \tilde{\lambda}(\pi(\tilde{N}))=\tilde{\lambda}\left(\pi^{-1}(\pi(\tilde{N}))\right) \geq \tilde{\lambda}(\tilde{N})=1$.

Applying the Ergodic Decomposition Theorem to $\lambda$ and using the affine dependence of the measure theoretic entropy on the measure (see formula S.2.5, 
p. 670 of [KH95]) an ergodic Bpm $\mu$ on $X$ with the desired properties is obtained.

\section{Proof of Theorem 3}

Recall that $f$ is continuum-expansive if there is $\varepsilon>0$ such that for every non-degenerate continuum $C$ there is $n \in \mathbb{Z}$ such that diam $f^{n}(C) \geq \varepsilon$. In what follows this $\varepsilon$ is kept fixed. The following convexity lemma will be needed:

Lemma 12. There exists $r>0$ such that, for every continuum $C$ and every integer $N>0$ satisfying $\operatorname{diam}(C)<r$ and $\operatorname{diam}\left(f^{N}(C)\right)<r$, it follows that $\operatorname{diam}\left(f^{n}(C)\right)<\varepsilon$ for every $0 \leq n \leq N$.

Proof. Let $B_{N}(x, \varepsilon)$ denote the set of points $y$ such that $d\left(f^{j}(x), f^{j}(y)\right) \leq \varepsilon$ for every $0 \leq j \leq N$. If the lemma is false, there are a sequence of continua $\Gamma_{n}$ and a sequence of integers $N_{n}$ such that $\operatorname{diam}\left(\Gamma_{n}\right)<1 / n, \operatorname{diam}\left(f^{N_{n}}\left(\Gamma_{n}\right)\right)<1 / n$ and such that there exist $0<j_{n}<N_{n}$ for which $\operatorname{diam}\left(f^{j_{n}}\left(\Gamma_{n}\right)\right) \geq \varepsilon$.

Choose points $x_{n} \in \Gamma_{n}$ and let $C_{n}$ be the connected component of the set $B_{N_{n}}\left(x_{n}, \varepsilon / 2\right) \cap \Gamma_{n}$ containing $x_{n}$. Then, $\operatorname{diam}\left(f^{i}\left(C_{n}\right)\right) \leq \varepsilon$ for every $0 \leq i \leq N_{n}$ and there is $0 \leq m_{n} \leq N_{n}$ such that $\operatorname{diam}\left(f^{m_{n}}\left(C_{n}\right)\right) \geq \varepsilon / 2$. Because $f$ is a homeomorphism and $M$ is compact, it follows that $m_{n} \rightarrow \infty$ and $N_{n}-m_{n} \rightarrow \infty$.

Set $D_{n}=f^{m_{n}}\left(C_{n}\right)$ and let $n_{k} \rightarrow \infty$ be a sequence of integers such that $D_{n_{k}}$ converges in the Hausdorff topology to the continuum $D$. Observe that $\operatorname{diam}(D) \geq \varepsilon / 2$. On the other hand, since $\operatorname{diam}\left(f^{i}\left(D_{n_{k}}\right)\right) \leq \varepsilon$ for $-m_{n_{k}} \leq i \leq$ $N_{n_{k}}-m_{n_{k}}$, we get $\operatorname{diam}\left(f^{i}(D)\right) \leq \varepsilon$ for every $i \in \mathbb{Z}$, contradicting continuumexpansivity.

Proof of Theorem 3. Take $5 \rho<r<\varepsilon$ where $r$ and $\varepsilon$ are as in Lemma 12. By continuum-expansivity and compactness there is $N$ such that for every continuum $C$ such that $\operatorname{diam}(C) \geq \rho$ there is $|m| \leq N$ such that $\operatorname{diam}\left(f^{m}(C)\right) \geq \varepsilon$.

Let $C$ be a continuum with $\operatorname{diam}(C)>\rho$ and assume there is $0 \leq m \leq N$ such that $\operatorname{diam}\left(f^{m}(C)\right) \geq \varepsilon$. Lemma 12 implies $\operatorname{diam}\left(f^{N}(C)\right)>r>5 \rho$ so it is possible to choose continua $C_{1,1}, C_{1,2} \subset f^{N}(C)$ such that $\rho<\operatorname{diam}\left(C_{1, i}\right)<2 \rho$ for $i=1,2$ and $d\left(C_{1,1}, C_{1,2}\right)>\rho$ (where $\left.d(A, B)=\inf \{d(a, b) \mid a \in A, b \in B\}\right)$. By induction on $k$ we construct continua $C_{k, i}, 1 \leq i \leq 2^{k}$ such that $\rho<\operatorname{diam}\left(C_{k, i}\right)<$ $2 \rho, d\left(C_{k, 2 i-1}, C_{k, 2 i}\right)>\rho, f^{-N}\left(C_{k, 2 i-1}\right) \subset C_{k-1, i}$ and $f^{-N}\left(C_{k, 2 i}\right) \subset C_{k-1, i}$. Assume that $C_{k, i}$ was constructed. We are going to construct $C_{k+1,2 i-1}$ and $C_{k+1,2 i}$. By continuum-expansivity there is $|m| \leq N$ such that $\operatorname{diam}\left(f^{m}\left(C_{k, i}\right)\right)>$ $\varepsilon$. It follows that $m>0$ because otherwise $\operatorname{diam}\left(f^{-N}\left(C_{k, i}\right)\right)>r>5 \rho$ and $\operatorname{diam}\left(C_{k, i}\right)>r>5 \rho$ by Lemma 12 and this, in turn, contradicts the properties of $C_{k, i}$. Applying Lemma 12 again it follows that $\operatorname{diam}\left(f^{N}\left(C_{k, i}\right)\right)>r>5 \rho$, which is enough to construct $C_{k+1,2 i-1}$ and $C_{k+1,2 i}$.

Let $T_{k}$ be a set obtained choosing exactly one point of each $C_{k, i}$ and set $S_{k}=f^{-k N}\left(T_{k}\right)$. Observe that the cardinality of $S_{k}$ is $2^{k}$. By construction, if $x, y \in S_{k}$ are different, there are $i \leq k$ and $j \leq 2^{i}$ such that $f^{i N}(x) \in C_{i, j}$ and 
$f^{i N}(y) \in C_{i, j+1}$ or vice versa. It follows that $d\left(f^{i N}(x), f^{i N}(y)\right)>\rho$, that is, $S_{k}$ is $(k N, \rho)$-separated. Therefore, $h(f, C)>0$.

An analogous argument holds with $f^{-1}$ in place of $f$ in case $-N \leq m \leq 0$. It follows that $h_{ \pm}(C)>0$ provided $\operatorname{diam}(C) \geq \rho$. This completes the proof because if $C$ is an arbitrary non-degenerate continuum, by continuum-expansivity there is $q$ such that $\operatorname{diam}\left(f^{q}(C)\right) \geq \varepsilon>\rho$.

The converse of Theorem 3 is not true in general. If $f$ is the cactoidal map obtained by gluing countably many copies of the tight horseshoe described in Section 3 at their fixed points, with diameters decreasing to 0 , then $f$ is tight but not continuum-expansive. We do not know whether the converse holds if attention is restricted to homeomorphisms of manifolds, for example.

We finish this section with a remark on the growth of lengths under tight maps. Given $\varepsilon>0$, define the $\varepsilon$-length $L_{\varepsilon}(\alpha)$ of a path $\alpha:[0,1] \rightarrow X$ as the smallest number $n$ for which it is possible to write $\alpha$ as the concatenation of $n$ paths $\alpha=\alpha_{1} \cdot \ldots \cdot \alpha_{n}$, with $\operatorname{diam}\left(\alpha_{i}\right)<\varepsilon$. It is not hard to show that tight maps expand the lengths of curves in the following sense: if $f: X \unlhd$ is a tight homeomorphism then for every path $\alpha:[0,1] \rightarrow X$ there is $\varepsilon>0$ such that $\lim \sup _{n \rightarrow \infty} \frac{1}{n} \ln L_{\varepsilon}\left(f^{n} \circ \alpha\right)>0$. If $f$ is continuum-expansive then the proof above shows that $\varepsilon$ can be chosen uniformly for every $\alpha$.

\section{Problems for further research}

There are several questions that arise naturally:

a) The Moore-Roberts-Steenrod theorems also give conditions under which the quotient space of a surface under a monotone upper semi-continuous decomposition is homeomorphic to the original surface. In the case of the sphere, for example, the condition is simply that the complement of every decomposition element be connected. It would be interesting to give conditions on the map to ensure that this is the case for the associated zero-entropy decomposition.

b) In the horseshoe example, the measure of maximal entropy gives rise to a Riemann surface structure on the quotient sphere together with an integrable quadratic differential which is meromorphic except for one essential singularity at the accumulation point of 1-pronged singularities (the poles of the quadratic differential). The quotient map is a Teichmüller mapping with respect to this structure. We believe similar statements hold whenever $f$ satisfies Axiom A and any two of its basic sets which do not reduce to periodic orbits are unrelated by Smale's partial order, that is, if $\Lambda, \Lambda^{\prime}$ are any two distinct basic sets, which are not periodic orbits, then $W^{s}(\Lambda) \cap W^{u}\left(\Lambda^{\prime}\right)=\emptyset$. This is related to a result of Bonatti-Jeandenans [BL98].

c) In work in preparation, the first author shows that, under certain assumptions, it is possible to take limits of sequences of generalized pseudo-Anosovs. The maps obtained are tight and the Riemann surface structure is still present, but the quadratic differentials may be only $L^{1}$ and have dense sets of 'zeros' and 'poles.' To find conditions under which the zero-entropy quotient 
of a surface diffeomorphism is the limit of generalized pseudo-Anosov maps seems an interesting problem. If this is the case, the quotient map should be thought of as an almost affine model for the diffeomorphism.

\section{Acknowledgements}

Both authors wish to thank the Institute for Mathematical Sciences of SUNY SB for the hospitality and support while this work was in progress.

\section{References}

[BL98] C. Bonatti and R. Langevin. Difféomorphismes de Smale des surfaces. Astérisque, 250 (1998), viii+235. With the collaboration of E. Jeandenans.

[Bow71] R. Bowen. Entropy for group endomorphisms and homogeneous spaces. Trans. Amer. Math. Soc., 153 (1971), 401-414.

[dC] A. de Carvalho. Extensions, quotients and generalized pseudo-anosov maps. ArXiv:math.DS/0204119. To appear.

[Gro87] M. Gromov. Entropy, homology and semialgebraic geometry. Astérisque, 145-146 (1987) 5, 225-240. Séminaire Bourbaki, Vol. 1985/86.

[Kat80] A. Katok. Lyapunov exponents, entropy and periodic orbits for diffeomorphisms. Inst. Hautes Études Sci. Publ. Math., 51 (1980), 137-173.

[KH95] A. Katok and B. Hasselblatt. Introduction to the modern theory of dynamical systems. Cambridge University Press, Cambridge, 1995. With a supplementary chapter by Katok and L. Mendoza.

[Mn87] R. Mañé. Ergodic theory and differentiable dynamics. Springer-Verlag, Berlin, 1987. Translated from the Portuguese by Silvio Levy.

[Moo62] R. Moore. Foundations of point set theory. American Mathematical Society, Providence, R.I., 1962.

[Pes76] Ja. B. Pesin. Families of invariant manifolds that correspond to nonzero characteristic exponents. Izv. Akad. Nauk SSSR Ser. Mat., 40 (1976) 6, 1332-1379, 1440.

[PS89] C. Pugh and M. Shub. Ergodic attractors. Trans. Amer. Math. Soc., 312 (1989) 1, $1-54$.

[RS38] J. Roberts and N. Steenrod. Monotone transformations of two-dimensional manifolds. Ann. of Math., 39 (1938), 851-862.

[Sma67] S. Smale. Differentiable dynamical systems. Bull. Amer. Math. Soc., 73 (1967), 747817.

Institute for Mathematical Sciences, SUNY, Stony Brook, NY 11794-3660

Current address: Departamento de Matemática Aplicada, IME - USP, Rua do Matão 1010, Cidade Universitária, 05508-090 São Paulo, SP, Brazil

E-mail address: andre@ime.usp.br

Centro de Matemática, Facultad de Ciencias, Eduardo Acevedo 1139, Montevideo CP 11200, URUGUAY

E-mail address: miguel@cmat.edu.uy 